\title{
5-Formylcytosine controls nucleosome positioning through covalent histone-DNA interaction
}

Eun-Ang Raiber ${ }^{1,10}$, Guillem Portella ${ }^{1,10}$, Sergio Martínez Cuesta ${ }^{1,2}$, Robyn Hardisty $^{1}$, Pierre Murat ${ }^{1}$, Zhe $\mathrm{Li}^{1}$, Mario lurlaro ${ }^{3,4}$, Wendy Dean ${ }^{3}$, Julia Spindel ${ }^{3}$, Dario Beraldi ${ }^{5}$, Mark A. Dawson ${ }^{6}$, Wolf Reik ${ }^{3,7}$, Shankar Balasubramanian ${ }^{1,2,8,9}$

${ }^{1}$ Department of Chemistry, University of Cambridge, Cambridge, UK

${ }^{2}$ Cancer Research UK Cambridge Institute, University of Cambridge, Cambridge, UK

${ }^{3}$ Epigenetics Programme, The Babraham Institute, Cambridge, UK

${ }^{4}$ Present address: Friedrich Miescher Institute for Biomedical Research, Basel, Switzerland

${ }^{5}$ Cancer Research Centre, Institute of Cancer Sciences, University of Glasgow, UK

${ }^{6}$ Cancer Research Division, Peter MacCallum Cancer Centre, University of Melbourne, Australia

${ }^{7}$ The Wellcome Trust Sanger Institute, Cambridge, UK

${ }^{8}$ School of Clinical Medicine, University of Cambridge, UK

${ }^{9}$ Correspondence should be addressed to S.B. (sb10031@cam.ac.uk)

${ }^{10}$ These authors have contributed equally to this work

Nucleosomes are the basic unit of chromatin that ensure genome integrity and control access to the genetic information. The organization of 
nucleosomes is influenced by the underlying DNA sequence itself, transcription factors or other transcriptional machinery associated proteins and chromatin remodeling complexes (1-4). Herein, we show that the naturally occurring DNA modification, 5-formylcytosine contributes to the positioning of nucleosomes. We show that the ability of $5 \mathrm{fC}$ to position nucleosomes in vitro is associated with the formation of covalent interactions between histone residues and $5 \mathrm{fC}$ in the form of Schiff bases. We demonstrate that similar interactions can occur in a cellular environment and define their specific genomic loci in mouse embryonic stem cells. Collectively, our findings identify $5 \mathrm{fC}$ as a determinant of nucleosomal organization in which $5 \mathrm{fC}$ plays a role in establishing distinct regulatory regions that are linked to gene expression Our study provides a previously unknown molecular mechanism, involving the formation of reversible-covalent bonds between chromatin and DNA that supports a molecular linkage between DNA sequence, DNA base modification and chromatin structure.

The organization of nucleosomes is an important feature to the chromatin structure. The DNA sequence itself has been shown to be a determinant of nucleosome positioning raising questions about the effect of DNA modifications on chromatin structure. 5 -Formylcytosine is generated by the oxidation of $5 \mathrm{mC}$ by TET enzymes. It can undergo base excision repair by the thymine DNA glycosylase (TDG) (5) and it is therefore thought to mark sites that undergo active demethylation (6). However, we have previously shown that the $5 \mathrm{fC}$ distribution 
was tissue-dependent in mice $(7)$ and that $5 \mathrm{fC}$ was a stable modification in genomic DNA in vivo (8). Additionally we and others have demonstrated that $5 \mathrm{fC}$ can alter the physical properties of the DNA double helix $(9,10)$ and the identification of 5fC-binding chromatin remodelers and transcription factors in vitro $(11,12)$ raises fundamental questions about its involvement in chromatin biology. Moreover, the nature of the formyl group confers specific chemical properties to $5 \mathrm{fC}$ that are unique from other cytosine modifications such as 5hydroxymethylcytosine $(5 \mathrm{hmC})$ and 5-carboxycytosine $(5 \mathrm{caC})$. We therefore hypothesized that $5 \mathrm{fC}$ may be important in nucleosomal organization, particularly during early development, where increased levels of $5 \mathrm{fC}$ have been reported (8). To test this hypothesis, we assessed the impact of $5 \mathrm{fC}$ on nucleosome core particles formation and stability. We carried out biochemical studies on three different DNA sequences, Widom 601, MMTV-A and 5SrDNA with varying CpG and GC content (Fig. 1A and Supplementary Table 1). All sequences are able to form nucleosomes of different stabilities and properties and have been extensively characterized (13-16). In addition, effects into 5-methylcytosine $(5 \mathrm{mC})(17,18)$ and 5-hydroxymethylcytosine $(5 \mathrm{hmC})$ (19) on nucleosome occupancy have been investigated on the Widom 601 sequence, however it is unclear how $5 \mathrm{fC}$ affects nucleosome formation and stability. To address this, we compared nucleosome occupancy after chaperone-mediated assembly using DNA with unmodified and modified cytosines. We observed that $5 \mathrm{mC}, 5 \mathrm{hmC}$ and $5 \mathrm{fC}$ each caused an increase in nucleosome occupancy compared to unmodified Widom 601 DNA (Fig. 1A), with 5fC-DNA significantly (unpaired t-test, $p$ value $\leq$ 0.0001 ) displaying the strongest effect (Fig. 1B). Notably we found that only $5 \mathrm{fC}$ 
consistently and significantly (unpaired t-test, $p$ value $\leq 0.01$ ) enhanced nucleosome occupancy on the MMTV-A and 5SrDNA sequences (Fig. 1B and Supplementary Fig. 1A and B).

Since the density of $5 \mathrm{fC}$ in the fully modified DNA sequences does not reflect densities observed within the genomic DNA context (20) we studied the effect of decreasing $5 \mathrm{fC}$ densities (100\% $-1 \% 5 \mathrm{fC}$ with $1 \% 5 \mathrm{fC} \sim 15 \mathrm{fC}$ per strand) on nucleosome formation. DNA with decreasing $5 \mathrm{fC}$ density generally caused increased nucleosome fractions for all three sequences, the only exception being a reduction in nucleosome fraction at $1 \% 5 \mathrm{fC}$ for the $5 \mathrm{SrDNA}$ sequence (Fig. 1C). This data was supported by our results obtained from reconstitution by competition dialysis (13) to determine differences in $\Delta \mathrm{G}$. Here our results showed that $1 \% 5 \mathrm{fC}$ DNA caused the most favorable shift in free energy change $\left(\Delta \Delta \mathrm{G}^{\circ}=\right.$ $-0.253 \pm 0.05 \mathrm{kcal} \mathrm{mol}^{-1}$ ) for nucleosome formation when referenced to unmodified DNA (Fig. 1D and Supplementary Fig. 1C). The capacity for DNA bending as required by nucleosome structure, is an important determinant to nucleosomal organization and it has been reported that a single $5 \mathrm{fC}$ unit significantly increases the flexibility of DNA (10), which may explain our observation.

The aldehyde functionality of $5 \mathrm{fC}$ is prone to nucleophilic addition (e.g. by the $\varepsilon$ amino group of lysine) suggesting a chemical mechanism for $5 \mathrm{fC}$ to promote nucleosome occupancy. It has recently been suggested that $5 \mathrm{fC}$ can form a covalent bond with proteins $(21,22)$. The formation of reversible, covalent Schiff base (imine) bonding between the formyl group of $5 \mathrm{fC}$ with proximal lysine residues of histones would provide a selective, covalent mechanism for positioning nucleosomes even with a single $5 \mathrm{fC}$ per nucleosome (Fig. 1E). To 
explore this hypothesis, we employed the reducing agent $\mathrm{NaCNBH}_{3}$ on nucleosome core particles obtained from 5fC-containing Widom sequence to irreversibly trap any Schiff base after its formation, (Fig. 1F and Supplementary Fig. 1D). Denaturing gel electrophoresis showed the appearance of a new higher molecular weight band that was not observed in the cytosine containing control DNA (Fig. 1F). Also in the absence of $\mathrm{NaCNBH}_{3}$ or treatment of nucleosomes with $\mathrm{EtONH}_{2}$ prior reduction (as a competitor to lysine residues) we did not observe a higher molecular weight band, consistent with reversible Schiff base formation between 5fC-DNA and histone proteins (Supplementary Fig. 1E). Proteomics mass spectrometry analysis of the higher molecular weight band from the gel identified the presence of histone core proteins $\mathrm{H} 2 \mathrm{~B}, \mathrm{H} 3$ and $\mathrm{H} 4$ in the complex, consistent with covalent DNA adduct formation with these histones (Supplementary Table 2). We then exploited polymerase-stalling, caused by the stable crosslink formed after $\mathrm{NaCNBH}_{3}$ reduction, using a single primer extension reaction followed by sequencing to identify specific $5 f C$-lysine interactions (Fig. $1 \mathrm{G}$ and Supplementary Fig. 1F). Particularly, we observed significant stalling sites where $5 \mathrm{fC}$ was in a $\mathrm{CpG}$ context and in the major groove of DNA facing the histone core demonstrating that formylation of $\mathrm{CpG}$ sites, rather than $\mathrm{C}$ residues, enhanced covalent interactions between histone proteins to the DNA sequence (Fig. $1 \mathrm{H}$ and Supplementary Fig. 1G). (3). It is also noteworthy that significant stalling sites were enriched in the vicinity of the nucleosome dyad. To identify the most probable interactions between histone residues and $5 \mathrm{fC}$, we associated 5fC-induced polymerase stalling sites with the most proximal (within $5 \AA$ ) lysinenucleotide distances computed from molecular dynamics simulations that model 
the Widom nucleosome (Fig. 1I and Supplementary Fig. 1G). We observed that lysine residues in close proximity to significant stalling sites in the dyad region were mostly found in the $\mathrm{H} 3$ protein (Fig. 1X and Table 1). These data suggest a structural model whereby specific interactions between $5 \mathrm{fC}$ and lysine residues from the $\mathrm{H} 3$ protein may contribute to nucleosome positioning.

Since the intrinsic sequence preference of nucleosomes itself is a known determinant for the nucleosomal organization $(3,23)$, we next asked whether 5fC-associated preference for nucleosomes had an impact on DNA sequencedirected nucleosome positioning. We generated four indexed libraries of unmodified or fully modified DNA sequences by sonication of the genomic DNA, adapter ligation and subsequent PCR amplification using either dCtp, d5mCtp, d5hmCtp or d5fCtp (Fig. 2A, see Method). These libraries of oligonucleotides were then pooled $(1: 1: 1: 1)$ and subsequently used in a nucleosome reconstitution assay where the total amount of DNA was in excess compared to histone proteins to select for higher affinity DNA sequences. Nucleosomes were separated from free DNA on a non-denaturing gel, followed by extraction of the nucleosome fraction. Sequencing of the input DNA pool (before nucleosome reconstitution) and the nucleosome fraction allowed the identification of significantly ( $p$-value $\leq 0.00001$ ) enriched nucleosomal DNA sequences from each library. We identified 2,977 sequences for cytosine, 884 for $5 \mathrm{mC}, 10,537$ for $5 \mathrm{hmC}$ and 2,481 for $5 \mathrm{fC}$ DNA libraries. Fig. $2 \mathrm{~B}$ shows the nucleosome enrichment (nucleosome reads/ reads of the corresponding sequence in the input) for the different DNA libraries revealing that overall $5 f C$ was significantly 
(two-sided Mann-Whitney test, $\mathrm{p}$ value $\leq 0.0001$ ) associated with the highest enrichment compared to unmodified and other cytosine modifications. Our study also revealed that positions of $5 \mathrm{fC}$-associated nucleosomes overlapped the least with nucleosomes associated with cytosine, $5 \mathrm{mC}$ or $5 \mathrm{hmC}$ demonstrating the ability for $5 f C$ DNA to uniquely position nucleosomes (Fig. 2C). When we calculated the actual fraction of overlapping bases (Fig. 2D) between all libraries (vertical $\cap$ horizontal), we observed that over $80 \%$ of the $5 \mathrm{mC}$-associated nucleosome positions overlapped with cytosine and 5hmC nucleosomes, whereas $5 \mathrm{fC}$ (and $5 \mathrm{hmC}$ ) only shared $20-30 \%$ of nucleosome positions between modifications. This observation is depicted in Fig. 2E, where MNase signals were enriched either in the $5 \mathrm{fC}$ or cytosine $/ 5 \mathrm{mC}$ nucleosomes at two representative genomic loci. We next analysed the GC content and CpG density associated with the DNA sequences enriched in nucleosomes. We found that cytosine and 5mCassociated nucleosome enrichment was largely independent of GC content (Fig. $2 \mathrm{~F})$. In contrast, 5fC- associated enrichment was influenced by low GC content. Similarly, lower $\mathrm{CpG}$ density was associated with higher enrichment of $5 \mathrm{fC}$ associated nucleosomes (Fig. G) demonstrating some interplay between DNA sequence context and DNA modifications on nucleosome formation in vitro. Taken together, these data show that formylation of DNA sequences with low GC and CpG density uniquely enhances nucleosome affinity.

We next investigated the effects of $5 f C$ within its natural sequence context, i. e. mainly within CpGs context, and density on nucleosome formation. We therefore used endogenous mouse embryonic brain genomic DNA to assemble nucleosomes and subsequently performed MNase-sequencing. Fig. $2 \mathrm{H}$ (left 
panel) represents normalized MNase reads at $5 \mathrm{fC}$ sites previously identified in hindbrain ( 7 ) and $2 \mathrm{~kb}$ up- and downstream of these sites. Our data revealed that natural $5 \mathrm{fC}$ sites are characterized by increased nucleosome density, as judged nucleosome enrichment, consistent with our biochemical observations using synthetic DNA model templates. In contrast, non-5fC sites with similar GC content did not show any enrichment in nucleosome density (Fig. $1 \mathrm{H}$, right panel). To demonstrate that the observed impact of $5 \mathrm{fC}$ on nucleosome organization was independent of DNA sequence preference of nucleosomes, we showed that removal of $5 \mathrm{fC}$ by generating genomic DNA only containing cytosines (by "erasing" 5fC through PCR amplification) resulted in the depletion of nucleosomes at the same $5 \mathrm{fC}$ sites (Fig. $1 \mathrm{H}$, left panel). Collectively, our data show the ability of $5 \mathrm{fC}$ to position nucleosomes within the genomic DNA sequence context, both at unnatural (and fully modified) and at naturally (low $5 \mathrm{fC}$ density) occurring $5 f C$ genomic loci.

Since $5 \mathrm{fC}$ distribution is tissue-specific (7) and its level is dynamic throughout development (8) it raises the question if the ability of $5 \mathrm{fC}$ to position nucleosomes is involved in the tissue-specific nucleosomal organization. We therefore generated genome-wide nucleosome maps in hindbrain and heart (E11.5) using MNase-seq to investigate the impact of $5 \mathrm{fC}$ on nucleosome organization in vivo. We observed clear nucleosome positioning centred at $5 \mathrm{fC}$ sites $(7)$ in both, hindbrain and heart tissues (Fig. 3A), consistent with our in vitro observations. Our study also showed that on average nucleosome occupancy at $5 \mathrm{fC}$ sites was significantly higher ( $p$-value $\leq 0.0001$, two-sided Mann-Whitney U-test) than at all 
other nucleosome positions, both in heart and hindbrain, demonstrating an intrinsic $5 \mathrm{fC}$ DNA preference of nucleosomes that support a role for $5 \mathrm{fC}$ in determining the organization of nucleosomes in vivo (Supplementary Fig. 2A).

When we looked into the sequence context of naturally occurring $5 \mathrm{fC}$ sites in brain, we found that increasing $5 \mathrm{fC}$ levels were associated with increasing $\mathrm{CpG}$ density (Supplementary Fig. 2C). Nucleosomes are generally found to be depleted at $\mathrm{CpG}$ dense regions such as $\mathrm{CpG}$ islands (CGIs) in vivo, with a clear link between CpG content and nucleosome depletion $(24,25)$. Notably, we observed that 5fC-containing CGls were actually enriched in nucleosomes compared to CGIs that lack $5 f C$. We also found that in contrast to non-5fC CGIs, mainly found at promoters, the subset of 5fC-CGls associated with nucleosomes was located in gene bodies of actively transcribed genes fundamental for brain development including Zfp238 (26), Tcf3 (27), Mycn (28), Foxp4 (29), Spen (30) and Mid1 (31), highlighting the importance of $5 \mathrm{fC}$ in the establishment of distinct nucleosomal organization at tissue-specific active genes. Given our previous observation that $5 \mathrm{fC}$ was enriched $(7)$ at sites marked by $\mathrm{H} 3 \mathrm{~K} 27 \mathrm{ac}$ and H3K4me1, both hallmarks of active enhancers, we next investigated the effect of $5 \mathrm{fC}$ on chromatin structure at these sites. For both, H3K27ac and H3K4me1, we observed higher nucleosome density in the presence of $5 \mathrm{fC}$ compared to non $5 \mathrm{fC}$ sites revealing a differential nucleosomal landscape at regulatory loci containing 5fC (Fig. 3F and G). Enhancers and promoters marked by histone modifications including H3K4me1 and H3K27ac are generally nucleosome depleted (4). In contrast, some active tissue-specific enhancers, characterized by relatively high nucleosome occupancy and accessibility that bind pioneer transcription factors to 
nucleosomal DNA, are believed to stimulate transcription $(32,33)$. Taken together our data support a model where $5 \mathrm{fC}$ is involved in the organization of nucleosomes at regulatory regions important for brain-specific activity. This hypothesis would suggest that $5 \mathrm{fC}$-associated nucleosome positioning could be linked to enhanced, brain-specific gene expression. To evaluate this, we compared the expression of all genes linked to predicted enhancers (34) with genes linked to predicted enhancers containing 5fC-associated nucleosomes (Fig. $3 \mathrm{H})$. We found that genes linked to $5 \mathrm{fC}$-nucleosome enhancers were indeed significantly more highly expressed ( $p$-value $\leq 0.0001$, two-sided Mann-Whitney U-test) supporting a role of $5 \mathrm{fC}$ in the organization of nucleosomes at regulatory sites that is important for gene regulation.

To further test this hypothesis, we used the TDG KO model to study the consequences of $5 \mathrm{fC}$ redistribution. The DNA repair enzyme TDG can excise $5 \mathrm{fC}$ and $5 \mathrm{caC}(5)$, and consequently genetic deletion of TDG results in the increase of $5 \mathrm{fC}$ levels with up to 10 -fold enhancement of $5 \mathrm{fC}$ sites genome-wide ( 7 ) concomitant with "new" $5 \mathrm{fC}$ sites in embryonic hindbrain and heart tissues at embryonic day 11.5 (E11.5). When we examined the consequences of TDGdependent enrichment of $5 \mathrm{fC}$ sites on the nucleosomal organization, we observed positioning of nucleosomes centered at $5 \mathrm{fC}$ sites in hindbrain and heart, similar to wild type, whereas nucleosomes were depleted at sites that lacked $5 f C$ sites (Fig. $3 \mathrm{I}$ and $3 \mathrm{~J}$ ). When we analysed the differential nucleosome occupancy between WT and TDG-KO we found significantly higher nucleosome occupancy ( $p$-value $<0.0001$, two-sided Mann-Whitney U-test) at sites where $5 \mathrm{fC}$ 
had been acquired, both in hindbrain and heart (Fig. 3K). To investigate a potential link between differential nucleosomal organization at emergent $5 \mathrm{fC}$ sites in the TDG KO and changes in gene expression, we considered differential expression of genes for which there was information on their predicted enhancers (Fig. 3L). Increased gene expression ( $p$-value $\leq 0.01$, two-sided Mann-Whitney U-test) was observed for genes where differential 5fC-associated nucleosomes aligned at the enhancers. Finally, we compared tissue-specific gene expression in WT and TDG KO tissues. We observed that particularly in WT hindbrain, differential gene expression (hindbrain versus heart) was significantly higher ( $p$ value $\leq 0.05$, two-sided Mann-Whitney U-test) for genes linked to differential $5 \mathrm{fC}$ nucleosomes enhancers (present in hindbrain but not heart) (Supplementary Fig. 2D). Notably, in the TDG KO we found that many more differential 5fCnucleosomes now overlapped with enhancers, 761 in heart and 422 hindbrain, that were also linked to significant ( $p$-value $<0.0001$, two-sided Mann-Whitney Utest) differential gene expression of their associated genes (Fig. 3M) demonstrating the importance of $5 \mathrm{fC}$-associated nucleosome organization.

Now that we have shown that $5 \mathrm{fC}$ can interact with histone residues via the formation of Schiff base and that $5 \mathrm{fC}$-associated nucleosome positioning at regulatory sites in vivo was important, we next evaluated whether this covalent $5 \mathrm{fC}$ and histone interactions may provide a molecular mechanism in native chromatin context. We therefore chemically trapped (using $\mathrm{NaCNBH}_{3}$ ) any Schiff base formed in nuclei from TDG KO mESC that contain relatively high levels of $5 f C$ (35). Subsequent covalent histone-DNA complexes were isolated by histone 
chromatin immunoprecipitation (ChIP) using four different antibodies to $\mathrm{H} 1, \mathrm{H} 2 \mathrm{~A}$, H3 and actin (Fig. 4A). Non-covalent DNA/ protein interactions were disrupted by guanidine $\mathrm{HCl}$ treatment followed by extensive washing through a filter column. We assessed the enrichment of DNA libraries obtained from ChIP using the reduced ( $\mathrm{NaCNBH}_{3}$-treated) over untreated sample by qPCR. We observed significant enrichment (t-test, $p$ value $\leq 0.01$ ) when the antibody for histone 3 (H3) was used, but not $\mathrm{H} 1$ or $\mathrm{H} 2 \mathrm{~A}$, suggesting covalent Schiff base formation between $5 \mathrm{fC}$ and $\mathrm{H} 3$ (Fig. 4B). We subsequently sequenced two biological replicates of the H3 ChIP libraries to characterize the sites of Schiff base formation. We found a total of 2,693 peaks (across two replicates) that were correlated with existing $5 \mathrm{fC}$ maps (36) to identify the distribution of covalently $5 \mathrm{fC}$-bound histones in the genome. We found 370 sites that overlapped with $5 \mathrm{fC}$ sites, half of which were found within genes (164 genes). Figure $4 \mathrm{C}$ shows a representative genomic locus, where $5 \mathrm{fC}$ and $\mathrm{H} 3$ sites overlaped within the gene body. To further determine functional consequences of $\mathrm{H} 3 / 5 \mathrm{fC}$ interactions within genes, we examined engaged Pol II sites using global nuclear run-on coupled with deep sequencing (GRO-seq) datasets (using datasets from Wang et al. (37)) of mESCs TDG KO. Metagene analysis of the Gro-Seq signals in mESCs TDG $\mathrm{KO}$ after 0, 10, 20 and 30 minutes of synchronized transcription showed slowing down of Pol II transcription elongation at genes where we have mapped $\mathrm{H} 3-5 \mathrm{fC}$ interaction sites ("crosslinked") compared to "all", 5fC (without crosslink) and $5 \mathrm{caC}$ containing genes with a new wave of transcription starting before $80 \mathrm{~kb}$ (Fig. 4 D). Notably, we found that crosslinked H3 sites were also enriched before $80 \mathrm{~kb}$. Finally, our analysis revealed an accumulation of transcriptionally engaged 
Pol II within $2 \mathrm{~kb}$ downstream from the center of all crosslinked $\mathrm{H} 3$ sites (Fig. 4E) suggesting involvement of covalent $5 \mathrm{fC}-\mathrm{H} 3$ interactions in transcription regulation.

In summary, we have shown that $5 \mathrm{fC}$ is a determinant of nucleosome organization in vitro and in vivo. Changes in $5 \mathrm{fC}$ patterns caused by loss of TDG (KO) led to nucleosome re-positioning and occupancy linked to changes in gene expression. We demonstrated in vitro that $5 \mathrm{fC}$ can form a covalent, reversible Schiff base within the nucleosome context that may explain the intrinsic preference of nucleosomes for 5fC DNA. Our data in mESCs provide evidence that $5 \mathrm{fC}$ and histone residues may also interact covalently in a cellular context. These 5fC-H3 sites may provide a stalling site for Pol II for the recruitment of proteins involved in transcription elongation or chromatin remodeling. Collectively our data support a model whereby $5 \mathrm{fC}$ contributes to the positioning of cell and tissue-specific nucleosomes providing a molecular mechanism to help explain how $5 \mathrm{fC}$ regulates gene expression during development and in the reinforcement of cell identity.

\section{Acknowledgments}

The Balasubramanian laboratory is supported by core funding from Cancer Research UK (C14303/A17197). SB is a Senior Investigator of the Wellcome Trust (grant no. 099232/z/12/z). ZL is supported by $A^{\star} S T A R$ (Singapore). The Reik laboratory is supported by BBSRC (BB/ K010867/1) and the Wellcome Trust (095645/Z/11/Z). 


\section{Figure legends}

Fig. 1. $5 f C$ enhances nucleosome occupancy and can form a Schiff base

\section{with histone residues}

A, Double-stranded DNA (Widom 601, MMTV-A and 5SrDNA) comprising unmodified or modified cytosines were used to reconstitute nucleosomes in the presence of NAP1. The table shows the length of the sequences and total number of modifiable cytosines $(\mathrm{xC})$ and number of $\mathrm{xC}$ within $\mathrm{CpG}$ dinucleotide context. B, Non-denaturing polyacrylamide gel shows the nucleosomal (Nucl.) and free DNA fraction of cytosine, $5 \mathrm{mC}, 5 \mathrm{hmC}$ and $5 \mathrm{fC}$ Widom DNA. C, Log2FC of the nucleosome fraction (Nucl./ total DNA) of cytosine DNA compared to $5 \mathrm{mC}$, 5hmC and 5fC DNA was plotted for the Widom (black), MMTV-A (turquoise) and 5SrDNA sequence (grey). The error bars represent the standard deviation from technical replicates $(n=3)$. Unpaired t test was used to calculate $p$ values $\left({ }^{* *} p \leq\right.$ $\left.0.01,{ }^{* * *} p \leq 0.0001\right)$. D, Nucleosomes were reconstituted using different $5 \mathrm{fC}$ density (1-100\%) Widom DNA. Normalized nucleosome fractions were plotted against $5 \mathrm{fC}$ density. The error bars represent the standard deviation from two technical replicates. E, Competitive DNA-histone reconstitution method was used for the measurement of quantitative free-energy of $5 \mathrm{fC}$ nucleosomes using the $5 \mathrm{~S}$ fragment DNA as competitor (4.2 $\mu \mathrm{g}$ and $100 \mathrm{ng}$ Widom). Differences in free energy changes $\left(\Delta \Delta \mathrm{G}^{\circ}\right)$ were obtained by subtracting the free energy change for the cytosine Widom DNA from the free energy change for each $5 \mathrm{fC}$ density Widom DNA and are represented as the mean along with the standard deviation from two replicates. F, 5fC-DNA forms a Schiff base with the primary amines of proximal lysines on histones within the nucleosome, which is then reduced to an 
irreversible, covalent crosslink using $\mathrm{NaCNBH}_{3}$. G, $12 \%$ Bis-Tris Protein gel electrophoresis was used to separate non-crosslinked from crosslinked nucleosomes. Two replicates are shown for each, cytosine and 5fC (both cy5labeled). The appearance of a second, higher DNA band was observed for $5 \mathrm{fC}$ DNA due to covalent DNA-protein complex that was submitted for mass spec analysis (Supplementary Table 2). H, DNA polymerase stalling sites were identified by sequencing to obtain the locations of crosslinked $5 \mathrm{fC}$ sites around the histone core. I, Upper panel: The $\log _{2}$ fold change between the number of reads stalling in a given position in the polymerase stop assay and the number of reads stalling in a untreated $5 \mathrm{fC}$ sample, both for the forward (orange) and reverse strand (blue). The gray sinusoidal line indicates the orientation of the major groove with respect to the histone core, ranging from 1 (histone-core facing) to -1 (facing away from the histone core). Significant (FDR value $<0.0001$, Benjamini-Hochberg correction on exact $p$-value from negative binomial distribution) stalling sites ( \pm 3 bases) around $\mathrm{CpG}$ dinucleotides within the DNA sequence are highlighted in grey. Lower panels: The closest lysines $(<5 \AA)$ in histone $2 \mathrm{~A}$ and $\mathrm{B}(\mathrm{H} 2 \mathrm{~A}, \mathrm{H} 2 \mathrm{~B})$, histone $3(\mathrm{H} 3)$ and histone $4(\mathrm{H} 4)$ facing the major grooves of the DNA pointing towards the histone core were identified. Based on the overlap of the computed data with stalling sites (dotted yellow and purple lines for forward and reverse strand) potential $5 \mathrm{fC}$ sites involved in the Schiff base formation were identified. J, Table summarizes findings from mass spec analysis (of the higher DNA band from gel), which was supported by data obtained from modeling. 
Fig. 2. 5fC within genomic sequence context enhances nucleosome positioning in vitro

A, A pool of DNA sequences containing either cytosine, $5 \mathrm{mC}, 5 \mathrm{hmC}$ or $5 \mathrm{fC}$ was generated by PCR using genomic DNA and used for subsequent nucleosome reconstitution and sequencing. B, Nucleosome enrichment (nucleosome library/input DNA library) for cytosine (blue), 5mC (orange), 5hmC (green) and 5fC (red) DNA was plotted. Notched boxplot shows the 1st, 2nd (median) and 3rd quartile, with whiskers extending to the minimum and maximum. P-values were obtained using the two-sided Mann-Whitney test ${ }^{* * * *} p$-value $\left.\leq 0.0001\right)$. C, Table shows the number of unique fraction of bases associated with cytosine, $5 \mathrm{mC}$, $5 \mathrm{hmC}$ or $5 \mathrm{fC}$ nucleosomes. D, Heatmap showing the fraction of overlapping bases when intersecting cytosine, $5 \mathrm{mC}, 5 \mathrm{hmC}$ and $5 \mathrm{fC}$-associated nucleosome sequences with each other. E, Representative genomic loci showing unique 5fCassociated nucleosome positioning (left panel, red) and overlap between cytosine (blue) and 5mC-associated (orange) nucleosome positioning (right panel). F, G, Heatmap showing sequence features linked to nucleosome enrichment. $\mathbf{H}$, MNase reads (normalized RPKM) of reconstituted nucleosomes using genomic DNA extracted from mouse embryonic hindbrain (11.5 days) show enrichment around $5 f C$ brain sites (left panel, blue line (WT)). MNase signals around 1) non$5 \mathrm{fC}$ sites (right panel, blue line) with comparable GC content (green line) and around 2) $5 \mathrm{fC}$ sites when genomic DNA containing only cytosine was used for reconstitution (WT PCR C only, left panel, orange line) show depletion of MNase signals. 
Fig. 3. $5 f C$ is a determinant of nucleosomal organization in vivo that is linked to gene expression

A, Normalized MNase signal was plotted around $5 \mathrm{fC}$ sites (dotted black line) in hindbrain (blue) and heart (orange) revealing positioning of nucleosomes at the center of $5 f C$ sites. B, MNase signal (RPKM) around 5fC CGI (blue lines) were compared to non-5fC CGI (orange) in hindbrain showing that $5 \mathrm{fC}$ at CGls can dictate nucleosome enrichment. C, D, H3K27ac and H3K4me1 sites also show differential nucleosome density that is dependent on $5 \mathrm{fC}$. The figure illustrates the enrichment of nucleosomes at H3K27ac and H3Kme1 sites that overlap with $5 \mathrm{fC}$ sites. E, Comparison of gene expression (log RPKM) of a subset of enhancers marked by 5fC-nucleosomes ( $n=517$, light blue) and all enhancer sites ( $n=13286$, dark blue) in WT hindbrain. Notched boxplot show that the presence of $5 \mathrm{fC}$ at enhancer sites is correlated with significantly higher gene expression ( $p$-value $\leq 2 . e-8$, two-sided Mann-Whitney U-test) of their associated genes compared to all expressed genes (predicted enhancer-gene list from Shen et al, Nature 2007(35)). Boxplot shows 1st, 2nd (median) and 3rd quartile, with notches representing the confidence interval around the median and the black diamond the mean, and the whiskers indicate the reach of the data points beyond the 1st (Q1) and 3rd (Q3) quartile (e.g. Q1+1.5*(Q3-Q1)). F, G, Normalized MNase signal around $5 \mathrm{fC} \mathrm{KO}$ and non- $5 \mathrm{fC}$ sites with comparable $\mathrm{GC}$ content in hindbrain (light blue for $5 \mathrm{fC}$ and dark blue for non-5fC) and heart tissues (orange for $5 f C$ and red for non-5fC) show that $5 f C$ sites are enriched in nucleosomes also in $\mathrm{KO}$ tissues. $\mathbf{H}$, Notched boxplot shows nucleosome occupancy of differentially positioned nucleosomes in TDG KO hindbrain. Differentially 
positioned nucleosomes (TDG KO / WT) were identified by DANPOS (FDR < 0.05, Benjamini-Hochberg correction on Poisson p-value). Average nucleosomes occupancy of 5fC-associated differentially positioned nucleosomes ( $n=2196$ for brain and $n=9967$ for heart) was significantly ( $p$-value $<0.0001$, two-sided MannWhitney U-test) higher than all differentially positioned nucleosomes ( $n=357079$ for brain and $n=439541$ for heart). I, Notched boxplot show that $5 \mathrm{fC}$-associated nucleosomal reorganization at enhancer sites in TDG KO ( $n=40$ for brain and $n=$ 220 for heart) is linked with significantly increased gene expression in hindbrain (blue, p-value < 0.001, two-sided Mann-Whitney U-test) and heart (orange, $p$ value $<0.05$, two-sided Mann-Whitney U-test) tissue compared to all differentially expressed genes ( $n=87$ for brain and $n=188$ for heart). J, Differential expression analysis between hindbrain (blue) and heart (orange) tissues in TDG KO. Significantly higher ( $p$-value $<0.0001$, two-sided Mann-Whitney U-test) differential expression was observed between hindbrain and heart tissue of genes linked to enhancers containing $5 f C$-associated nucleosomes (for brain $n=$ 422 and for heart $n=761$ ) than all enhancers (for brain $n=1827$ and for heart $n=$ 2082). All boxplots in this figure show the 1st, 2nd (median) and 3rd quartiles, with notches representing the confidence interval around the median and the black diamond the mean, and the whiskers indicate the reach of the data points beyond the 1st (Q1) and 3rd (Q3) quartile (e.g. Q1+1.5*(Q3-Q1)).

Fig. 4. 5fC can form Schiff base with histones in chromatin context that impacts polymerase activity 
A, Workflow for the detection of in vivo Schiff base in mESC TDG KO. Key steps involve the reduction of the imine bond using $\mathrm{NaCNBH}_{3}$ followed by denaturation of proteins to disrupt any non-crosslinked DNA-protein interaction and subsequent histone ChIP, with no reduction for the control. B, Log2FC of reduced/untreated samples after ChIP-qPCR reveal significant enrichment (t-test, ** $p$-value $\leq 0.01$ ) of H3 immunoprecipitated chromatin after reduction. Scatter dot plot show the values for individual replicates for actin (blue), H1 (orange), $\mathrm{H} 2 \mathrm{~A}$ (green) and $\mathrm{H} 3$ (red) with lines indicating the mean with standard deviation. C, Representative genomic loci showing the overlap between $5 \mathrm{fC}$ and $5 \mathrm{fC} / \mathrm{H} 3$ sites in mESC TDG KO. D, Metagene analysis of normalized Gro-Seq signal at genes containing All, 5fC, 5caC or crosslinked $\mathrm{H} 3$ sites at 0 (blue), 10 (orange), 20 (green) and 30 minutes (red) after DRB treatment. Bottom panel shows the distribution of crosslinked H3 coverage (RPKM) across the genes E, Gro-Seq signals (RPKM) centered around 25kb up- and downstream of $\mathrm{H} 3 / 5 \mathrm{fC}$ sites (green) and $5 \mathrm{fC}$ only (blue) sites. 
1. S. Venkatesh, J. L. Workman, Histone exchange, chromatin structure and the regulation of transcription. Nat. Rev. Mol. Cell Biol. 16, 178-189 (2015).

2. M. Radman-Livaja, O. J. Rando, Nucleosome positioning: How is it established, and why does it matter? Dev. Biol. 339, 258-266 (2010).

3. E. Segal et al., A genomic code for nucleosome positioning. Nature. 442, $772-778$ (2006).

4. K. Struhl, E. Segal, Determinants of nucleosome positioning. Nat. Struct. Mol. Biol. 20, 267-273 (2013).

5. A. Maiti, A. C. Drohat, Thymine DNA Glycosylase Can Rapidly Excise 5Formylcytosine and 5-Carboxylcytosine: POTENTIAL IMPLICATIONS FOR ACTIVE DEMETHYLATION OF CpG SITES. J. Biol. Chem. 286, 35334-35338 (2011).

6. X. Wu, Y. Zhang, TET-mediated active DNA demethylation: mechanism, function and beyond. Nat. Rev. Genet. (2017), doi:10.1038/nrg.2017.33.

7. M. Iurlaro et al., In vivo genome-wide profiling reveals a tissue-specific role for 5-formylcytosine. Genome Biol. 17, 141 (2016).

8. M. Bachman et al., 5-Formylcytosine can be a stable DNA modification in mammals. Nat. Chem. Biol. 11, 555-557 (2015).

9. E.-A. Raiber et al., 5-Formylcytosine alters the structure of the DNA double helix. Nat. Struct. Mol. Biol. 22, 44-49 (2014).

10. T. T. M. Ngo et al., Effects of cytosine modifications on DNA flexibility and nucleosome mechanical stability. Nat. Commun. 7, 10813 (2016).

11. M. Iurlaro et al., A screen for hydroxymethylcytosine and formylcytosine binding proteins suggests functions in transcription and chromatin regulation. Genome Biol. 14, R119 (2013). 
12. C. G. Spruijt et al., Dynamic Readers for 5-(Hydroxy)Methylcytosine and Its Oxidized Derivatives. Cell. 152, 1146-1159 (2013).

13. A. Thåström, P. . Lowary, J. Widom, Measurement of histone-DNA interaction free energy in nucleosomes. Methods. 33, 33-44 (2004).

14. P. . Lowary, J. Widom, New DNA sequence rules for high affinity binding to histone octamer and sequence-directed nucleosome positioning. J. Mol. Biol. 276, 19-42 (1998).

15. F. Dong, J. C. Hansen, K. E. van Holde, DNA and protein determinants of nucleosome positioning on sea urchin 5S rRNA gene sequences in vitro. Proc. Natl. Acad. Sci. 87, 5724-5728 (1990).

16. T. D. Frouws, S. C. Duda, T. J. Richmond, X-ray structure of the MMTV-A nucleosome core. Proc. Natl. Acad. Sci. 113, 1214-1219 (2016).

17. J. S. Choy et al., DNA methylation increases nucleosome compaction and rigidity. J. Am. Chem. Soc. 132, 1782-3 (2010).

18. J. Y. Lee, T.-H. Lee, Effects of DNA methylation on the structure of nucleosomes. J. Am. Chem. Soc. 134, 173-5 (2012).

19. S. Kizaki, Y. Suzuki, T. Takenaka, M. Endo, H. Sugiyama, AFM analysis of changes in nucleosome wrapping induced by DNA epigenetic modification. Biomater. Sci. 2, 1399 (2014).

20. M. J. Booth, G. Marsico, M. Bachman, D. Beraldi, S. Balasubramanian, Quantitative sequencing of 5-formylcytosine in DNA at single-base resolution. Nat. Chem. 6, 435-440 (2014).

21. S. Ji, H. Shao, Q. Han, C. L. Seiler, N. Y. Tretyakova, Reversible DNA-Protein Cross-Linking at Epigenetic DNA Marks. Angew. Chemie Int. Ed. 56, 1413014134 (2017). 
22. F. Li et al., 5-Formylcytosine Yields DNA-Protein Cross-Links in Nucleosome Core Particles. J. Am. Chem. Soc. 139, 10617-10620 (2017).

23. N. Kaplan et al., The DNA-encoded nucleosome organization of a eukaryotic genome. Nature. 458, 362-366 (2009).

24. A. Valouev et al., Determinants of nucleosome organization in primary human cells. Nature. 474, 516-520 (2011).

25. R. Fenouil et al., CpG islands and GC content dictate nucleosome depletion in a transcription-independent manner at mammalian promoters. Genome Res. 22, 2399-2408 (2012).

26. J. I.-T. Heng et al., The Zinc Finger Transcription Factor RP58 Negatively Regulates Rnd2 for the Control of Neuronal Migration During Cerebral Cortical Development. Cereb. Cortex. 25, 806-816 (2015).

27. T. Ohtsuka et al., Gene Expression Profiling of Neural Stem Cells and Identification of Regulators of Neural Differentiation During Cortical Development. Stem Cells. 29, 1817-1828 (2011).

28. P. S. Knoepfler, N-myc is essential during neurogenesis for the rapid expansion of progenitor cell populations and the inhibition of neuronal differentiation. Genes Dev. 16, 2699-2712 (2002).

29. K. Takahashi, F.-C. Liu, K. Hirokawa, H. Takahashi, Expression of Foxp4 in the developing and adult rat forebrain. J. Neurosci. Res. 86, 3106-3116 (2008).

30. B. Kuang, S. C. Wu, Y. Shin, L. Luo, P. Kolodziej, split ends encodes large nuclear proteins that regulate neuronal cell fate and axon extension in the Drosophila embryo. Development. 127, 1517-29 (2000).

31. T. Lu et al., X-linked microtubule-associated protein, Mid1, regulates axon 
development. Proc. Natl. Acad. Sci. 110, 19131-19136 (2013).

32. M. Iwafuchi-Doi et al., The Pioneer Transcription Factor FoxA Maintains an Accessible Nucleosome Configuration at Enhancers for Tissue-Specific Gene Activation. Mol. Cell. 62, 79-91 (2016).

33. J. Mieczkowski et al., MNase titration reveals differences between nucleosome occupancy and chromatin accessibility. Nat. Commun. 7, 11485 (2016).

34. Y. Shen et al., A map of the cis-regulatory sequences in the mouse genome. Nature. 488, 116-120 (2012).

35. C.-X. Song et al., Genome-wide Profiling of 5-Formylcytosine Reveals Its Roles in Epigenetic Priming. Cell. 153, 678-691 (2013).

36. L. Shen et al., Genome-wide Analysis Reveals TET- and TDG-Dependent 5Methylcytosine Oxidation Dynamics. Cell. 153, 692-706 (2013).

37. L. Wang et al., Molecular basis for 5-carboxycytosine recognition by RNA polymerase II elongation complex. Nature. 523, 621-625 (2015).

38. D. Cortázar et al., Embryonic lethal phenotype reveals a function of TDG in maintaining epigenetic stability. Nature. 470, 419-423 (2011).

39. B. Hess, C. Kutzner, D. van der Spoel, E. Lindahl, GROMACS 4: Algorithms for Highly Efficient, Load-Balanced, and Scalable Molecular Simulation. J. Chem. Theory Comput. 4, 435-447 (2008).

40. T. Darden, D. York, L. Pedersen, Particle mesh Ewald: An N $\cdot \log (\mathrm{N})$ method for Ewald sums in large systems. J. Chem. Phys. 98, 10089-10092 (1993).

41. S. Miyamoto, P. A. Kollman, Settle: An analytical version of the SHAKE and RATTLE algorithm for rigid water models. J. Comput. Chem. 13, 952-962 
(1992).

42. B. Hess, P-LINCS: A Parallel Linear Constraint Solver for Molecular Simulation. J. Chem. Theory Comput. 4, 116-122 (2008).

43. K. A. Feenstra, B. Hess, H. J. C. Berendsen, Improving efficiency of large time-scale molecular dynamics simulations of hydrogen-rich systems. J. Comput. Chem. 20, 786-798 (1999).

44. G. Bussi, D. Donadio, M. Parrinello, Canonical sampling through velocity rescaling. J. Chem. Phys. 126, 14101 (2007).

45. H. J. C. Berendsen, J. P. M. Postma, W. F. van Gunsteren, A. DiNola, J. R. Haak, Molecular dynamics with coupling to an external bath. J. Chem. Phys. 81, 3684-3690 (1984).

46. R. B. Best, G. Hummer, Optimized Molecular Dynamics Force Fields Applied to the Helix-Coil Transition of Polypeptides. J. Phys. Chem. B. 113, 9004-9015 (2009).

47. A. Pérez et al., Refinement of the AMBER Force Field for Nucleic Acids: Improving the Description of $\alpha / \gamma$ Conformers. Biophys. J. 92, 3817-3829 (2007).

48. D. E. Smith, L. X. Dang, Computer simulations of $\mathrm{NaCl}$ association in polarizable water. J. Chem. Phys. 100, 3757-3766 (1994).

49. R. Collepardo-Guevara et al., Chromatin Unfolding by Epigenetic Modifications Explained by Dramatic Impairment of Internucleosome Interactions: A Multiscale Computational Study.J. Am. Chem. Soc. 137, 10205-10215 (2015).

50. C. A. Davey, D. F. Sargent, K. Luger, A. W. Maeder, T. J. Richmond, Solvent Mediated Interactions in the Structure of the Nucleosome Core Particle at 
1.9Å Resolution. J. Mol. Biol. 319, 1097-1113 (2002).

51. H. Li, R. Durbin, Fast and accurate short read alignment with BurrowsWheeler transform. Bioinformatics. 25, 1754-1760 (2009).

52. F. Ramírez et al., deepTools2: a next generation web server for deepsequencing data analysis. Nucleic Acids Res. 44, W160-W165 (2016).

53. Y. Zhang et al., Model-based Analysis of ChIP-Seq (MACS). Genome Biol. 9, R137 (2008).

54. W. Chen et al., Improved nucleosome-positioning algorithm iNPS for accurate nucleosome positioning from sequencing data. Nat. Commun. 5, 4909 (2014).

55. K. Chen et al., DANPOS: Dynamic analysis of nucleosome position and occupancy by sequencing. Genome Res. 23, 341-351 (2013). 
Fig. 1

A

\begin{tabular}{lccc}
\hline & $\begin{array}{c}\text { Length } \\
\text { (bp) }\end{array}$ & $\begin{array}{c}\text { Modifiable } \\
\text { xC }\end{array}$ & $\begin{array}{c}\text { xC within } \\
\text { CpG }\end{array}$ \\
\hline Widom & 147 & 67 & 10 \\
MMTV-A & 147 & 73 & 8 \\
5SrDNA & 208 & 86 & 7 \\
\hline
\end{tabular}

B

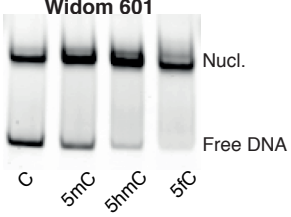

E

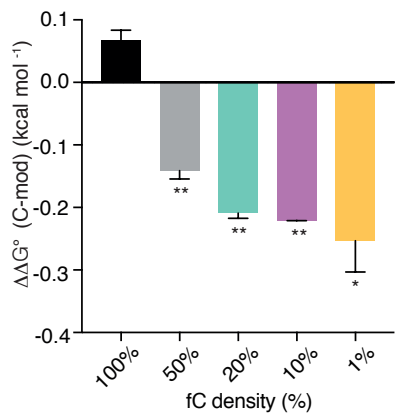

J

\begin{tabular}{ccc}
\hline $\begin{array}{c}\text { Detected } \\
\text { (Mass spec) }\end{array}$ & $\begin{array}{c}\text { Predicted } \\
\text { (Modeling) }\end{array}$ & $\begin{array}{c}\text { Predicted lys res } \\
\text { (Modeling) }\end{array}$ \\
\hline & H2A_1 Tail & 8,36 \\
H2B & H2B_1 Tail & $2,12,24$ \\
H3 & H3 & 23,115 \\
H4 & H4 & $11,16,31$ \\
\hline
\end{tabular}

C

D

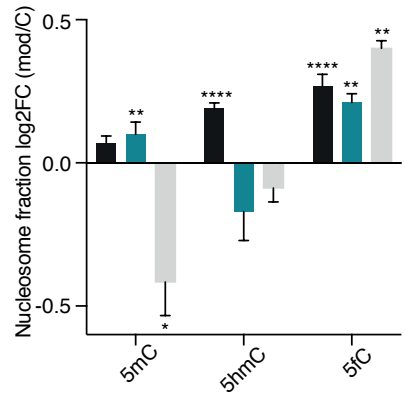

F

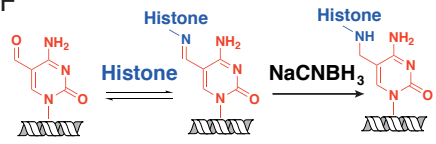

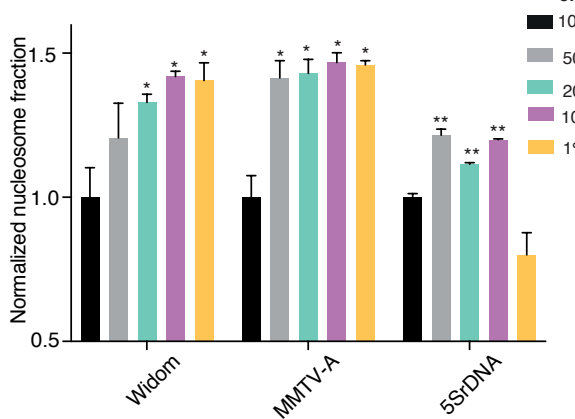

$\mathrm{H}$

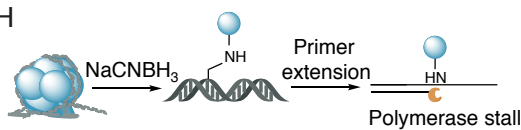

$5 f C$

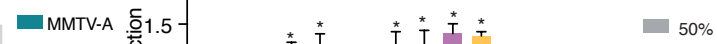

$10 \%$

更

Polymerase stall
G

$225 \mathrm{kDa}$

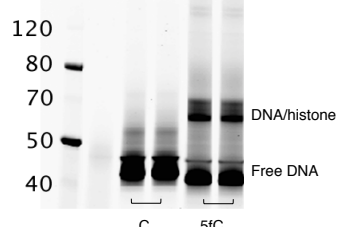

I
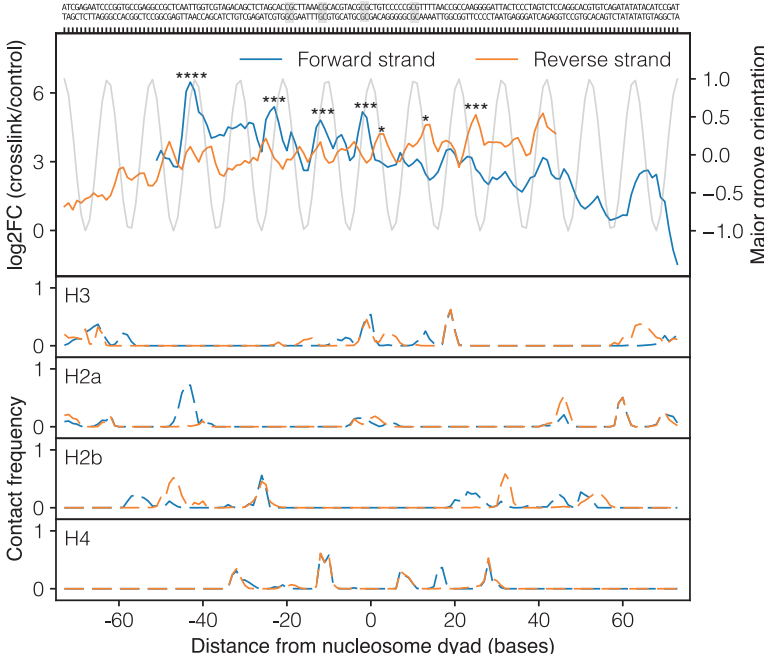
Fig. 3

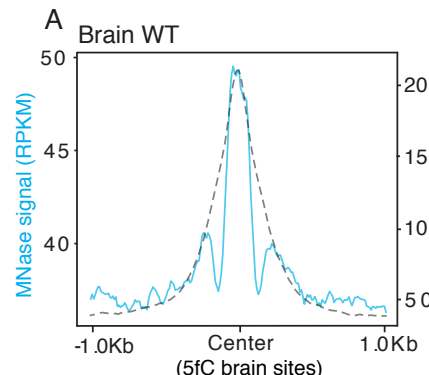

C

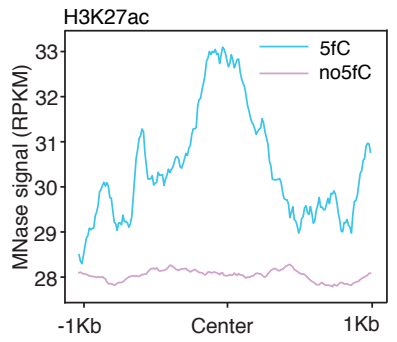

G

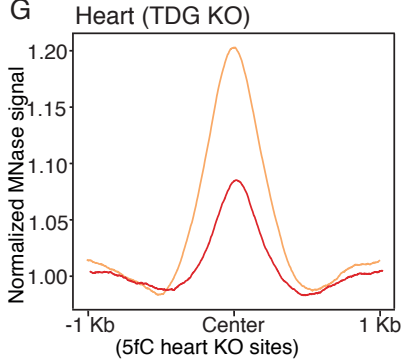

Heart WT

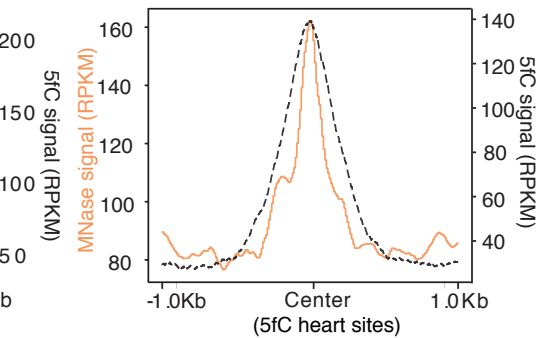

D

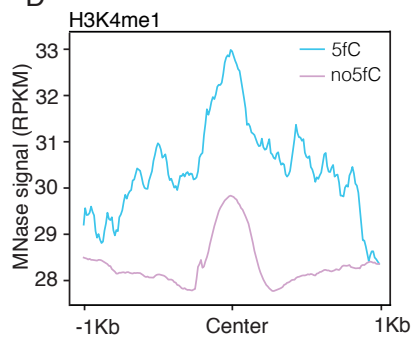

$\mathrm{E}$

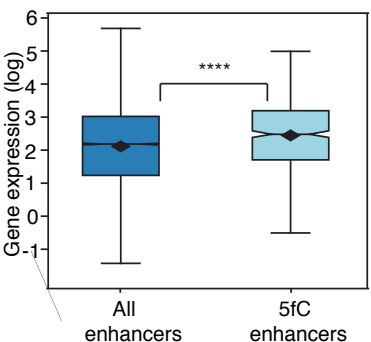

H TDG KO

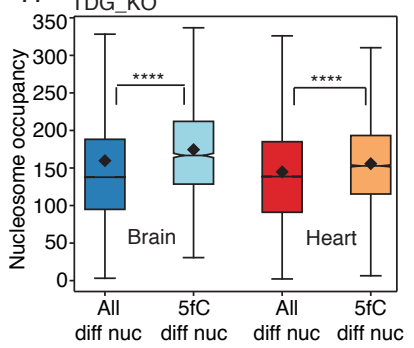

B

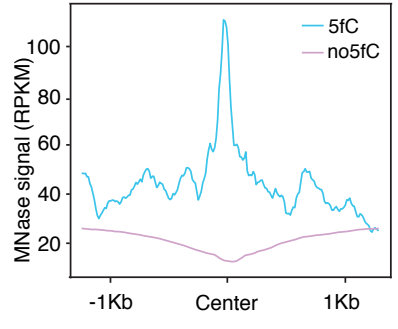

F Brain (TDG KO)

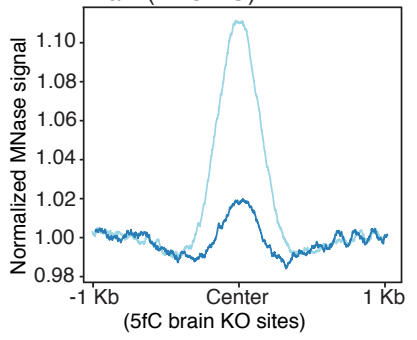

J TDG KO
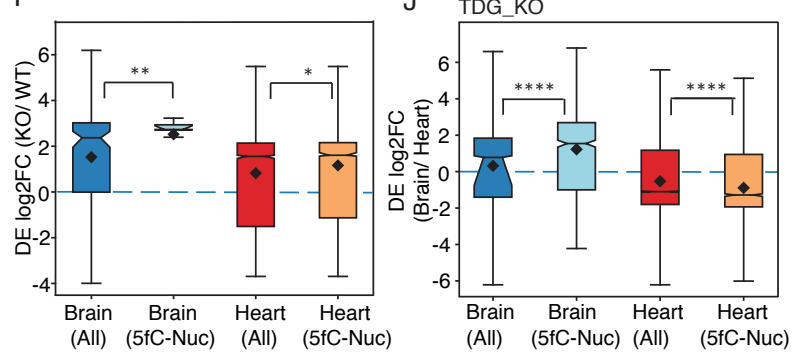
